\title{
Microcanonical rates from ring-polymer molecular dynamics: Direct-shooting, stationary-phase, and maximum-entropy approaches ${ }^{\circ}$
}

Cite as: J. Chem. Phys. 152, 124117 (2020); https://doi.org/10.1063/1.5144307

Submitted: 05 January 2020 . Accepted: 06 March 2020. Published Online: 30 March 2020

Xuecheng Tao, Philip Shushkov (D), and Thomas F. Miller (D)

\section{COLLECTIONS}

EP This paper was selected as an Editor's Pick

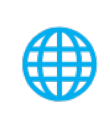

Lock-in Amplifiers Find out more today
Zurich

Instruments

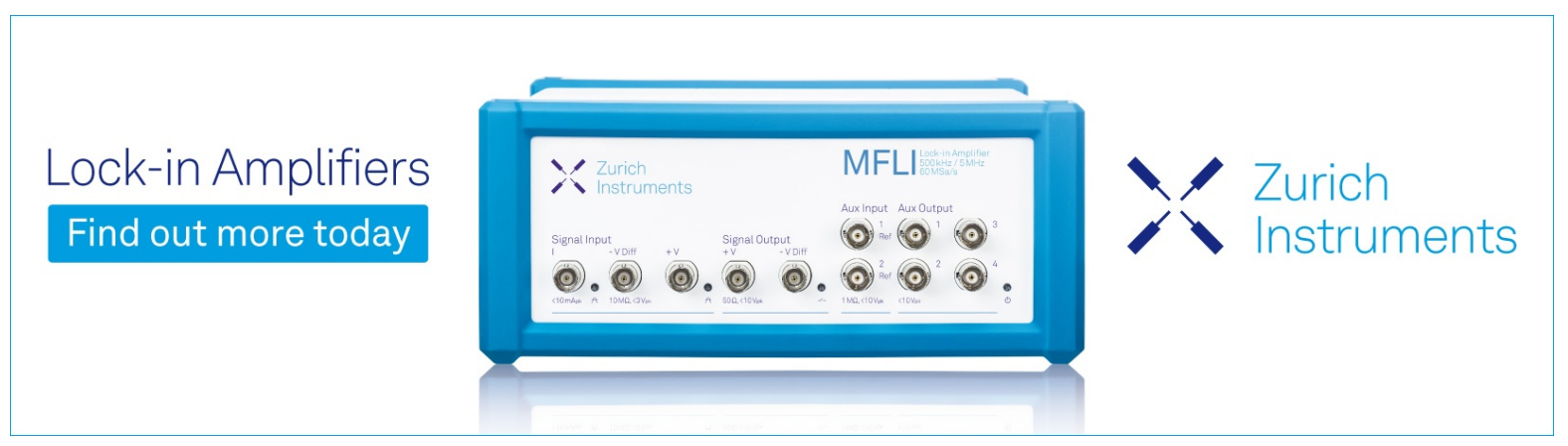




\title{
Microcanonical rates from ring-polymer molecular dynamics: Direct-shooting, stationary-phase, and maximum-entropy approaches 루
}

Cite as: J. Chem. Phys. 152, 124117 (2020); doi: 10.1063/1.5144307

Submitted: 5 January 2020 - Accepted: 6 March 2020 •

Published Online: 30 March 2020

Xuecheng Tao, Philip Shushkov, (D) and Thomas F. Miller III ${ }^{\text {a) }}$ (D)

\section{AFFILIATIONS}

Division of Chemistry and Chemical Engineering, California Institute of Technology, Pasadena, California 91125, USA

\author{
a) Author to whom correspondence should be addressed: tfm@caltech.edu
}

\begin{abstract}
We address the calculation of microcanonical reaction rates for processes involving significant nuclear quantum effects using ring-polymer molecular dynamics (RPMD), both with and without electronically non-adiabatic transitions. After illustrating the shortcomings of the naive free-particle direct-shooting method, in which the temperature of the internal ring-polymer modes is set to the translational energy scale, we investigate alternative strategies based on the expression for the microcanonical rate in terms of the inverse Laplace transform of the thermal reaction rate. It is shown that simple application of the stationary-phase approximation (SPA) dramatically improves the performance of the microcanonical rates using RPMD, particularly in the low-energy region where tunneling dominates. Using the SPA as a Bayesian prior, numerically exact RPMD microcanonical rates are then obtained using maximum entropy inversion of the thermal reaction rates for both electronically adiabatic and non-adiabatic model systems. Finally, the direct-shooting method is revisited using the SPA-determined temperature for the internal ring-polymer modes, leading to a simple, direct-simulation method with improved accuracy in the tunneling regime. This work suggests a general strategy for the extraction of microcanonical dynamical quantities from RPMD (or other approximate thermal) simulations.
\end{abstract}

Published under license by AIP Publishing. https://doi.org/10.1063/1.5144307

\section{INTRODUCTION}

Ring-polymer molecular dynamics (RPMD) $)^{1,2}$ has proven to be a useful tool for the calculation of chemical reaction rates, spectra, ${ }^{5,6}$ and transport coefficients. ${ }^{7,8}$ The method has been widely applied for the study of electronically adiabatic processes in which nuclear quantum effects play an important role, ${ }^{9-30}$ and extensions of the method for systems involving electronically non-adiabatic processes are increasingly common. ${ }^{31-42}$ However, despite the utility of RPMD for calculating quantities in terms of thermal transport coefficients, less work has focused on the extension of the method to non-thermal initial distributions ${ }^{43}$ or for the calculation of properties associated with non-thermal ensembles, such as microcanonical reaction rates, which would be of use for both benchmarking and practical applications.
Application of RPMD beyond the canonical ensemble immediately encounters the question of how to treat the temperature associated with the intra-bead ring-polymer potential. This temperature is well-defined in thermal applications for which RPMD was initially developed, ${ }^{1,2}$ and it has been justified for RPMD with particular non-equilibrium initial conditions. ${ }^{43}$ In the context of microcanonical reaction rates, a direct-shooting method based on the free-particle temperature has been proposed, ${ }^{44}$ in which the internal ring-polymer temperature is fixed based on the microcanonical energy, i.e., $T=E / k_{\mathrm{B}}$. This protocol has been employed in several model calculations, ${ }^{44,45}$ although its reliability has not been systematically examined.

This work addresses the challenge of microcanonical rate calculations using RPMD. In addition to analyzing the previously proposed free-particle direct-shooting protocol, we introduce 
alternative stationary-phase and maximum-entropy inversion methods to extract microcanonical rates from thermal reaction rates, the calculation of which is well established using RPMD. Finally, we return to the direct-shooting method for microcanonical RPMD rates, replacing the free-particle temperature with the optimal temperature from the stationary-phase inversion, which is shown to yield greatly improved microcanonical RPMD rates in the lowenergy regime. Numerical examples of these microcanonical RPMD methods are presented for both electronically adiabatic and nonadiabatic systems.

\section{METHODS}

\section{A. Thermal reaction rates from RPMD}

We begin by briefly reviewing RPMD and its use for the calculation of thermal reaction rates. The theory is presented for a one-dimensional system, and extension to multiple dimensions is straightforward. Consider an electronically adiabatic system with the Hamiltonian

$$
\hat{H}=\frac{\hat{p}^{2}}{2 m}+V(\hat{q}),
$$

where $V(\hat{q})$ is the potential energy function. Expressing the quantum canonical partition function, $Q$, in the path-integral representation yields ${ }^{46-48}$

$$
\begin{aligned}
Q & =\operatorname{tr}\left[e^{-\beta \hat{H}}\right] \\
& =\lim _{n \rightarrow \infty}\left(\frac{n}{2 \pi \hbar}\right)^{n} \int d \mathbf{p} d \mathbf{q} e^{-\beta H_{n}^{\text {iso }}(\mathbf{p}, \mathbf{q})},
\end{aligned}
$$

where $\beta$ and $n$ are the reciprocal temperature and the number of imaginary time discretization steps, respectively; $\mathbf{q}=\left\{q_{1}, q_{2}, \ldots, q_{n}\right\}$ denotes the positions of the ring-polymer beads, and $\mathbf{p}$ denotes the corresponding momenta. Equation (2) introduces the classical isomorphic ring-polymer Hamiltonian,

$$
H_{n}^{\mathrm{iso}}(\mathbf{p}, \mathbf{q})=\sum_{\alpha=1}^{n} \frac{p_{\alpha}^{2}}{2 m_{n}}+U_{\mathrm{spr}}(\mathbf{q})+\frac{1}{n} \sum_{\alpha=1}^{n} V\left(q_{\alpha}\right),
$$

where $\beta_{n}=\beta / n, m_{n}=m / n$, and neighboring ring-polymer beads are connected via harmonic springs

$$
U_{\text {spr }}(\mathbf{q})=\frac{1}{2} \frac{m_{n}}{\beta_{n}^{2}} \sum_{\alpha=1}^{n}\left(q_{\alpha}-q_{\alpha+1}\right)^{2} .
$$

Classical sampling of the ring-polymer Hamiltonian faithfully preserves quantum Boltzmann statistics. The classical equations of motion associated with the ring polymer Hamiltonian are given by

$$
\ddot{q}_{\alpha}=\frac{1}{\beta_{n}^{2}}\left(q_{\alpha+1}+q_{\alpha-1}-2 q_{\alpha}\right)-\frac{1}{m} \frac{\partial V\left(q_{\alpha}\right)}{\partial q_{\alpha}} .
$$

The calculation of thermal rates from RPMD then simply follows from the application of classical rate theory to the dynamics associated with the ring-polymer Hamiltonian. ${ }^{2-4}$ Specifically, calculation of the thermal RPMD rate in the flux-side formulation yields

$$
\begin{aligned}
k Q_{r}= & \lim _{n \rightarrow \infty} \lim _{t \rightarrow{ }^{\prime \prime}}\left(\frac{n}{2 \pi \hbar}\right)^{n} \int d \mathbf{p}_{0} d \mathbf{q}_{0} e^{-\beta H_{n}^{\text {iso }}\left(\mathbf{p}_{0}, \mathbf{q}_{0}\right)} \\
& \times \delta\left(\bar{q}_{0}-q^{\ddagger}\right) \bar{v}_{0} h\left(\bar{q}_{t}-q^{\ddagger}\right),
\end{aligned}
$$

which correlates the positions and velocities of the ring-polymer beads at time $t$ following evolution according to the ring-polymer equations of motion [Eq. (5)] from an initial distribution in which the ring-polymer centroid is positioned at the dividing surface for the reaction. Here, $\bar{q}_{0}$ and $\bar{q}_{t}$ indicate the ring-polymer centroid position at time zero and $t$, respectively, and $\bar{v}_{0}$ indicates the centroid velocity at time zero. $Q_{r}$ denotes the reactant partition function, $q^{\ddagger}$ indicates the position of the dividing surface that separates the reactant and product, $h$ is the Heaviside function, and $\delta$ is the Dirac delta function.

\section{B. Microcanonical reaction rates from RPMD}

Here, we describe three alternative strategies for calculating microcanonical reaction rates from RPMD. The first involves an inverse Laplace transform of the thermal RPMD reaction rates and introduces no approximations beyond that of the thermal RPMD rate theory, although it is numerically the most demanding. The subsequent two methods introduce additional approximations (i.e., the stationary phase approximation and the direct shooting approximation) with the benefit of reduced numerical complexity.

\section{Maximum entropy inversion}

Reaction rates in the microcanonical ${ }^{49}$ and canonical ensembles are connected via the Laplace transform ${ }^{50}$

$$
k(\beta) Q_{r}(\beta)=\frac{1}{2 \pi \hbar} \int_{-\infty}^{+\infty} d E e^{-\beta E} N(E)
$$

which can be inverted to yield ${ }^{51-53}$

$$
N(E)=(2 \pi \hbar) \frac{1}{2 \pi i} \int_{\gamma-i \infty}^{\gamma+i \infty} d \beta e^{\Phi(\beta)}
$$

where

$$
\Phi(\beta)=\beta E+\log \left(k Q_{r}\right) .
$$

The line integral in Eq. (8) is performed along $\operatorname{Re}[\beta]=\gamma$, where $\gamma$ is greater than the real part of all points for which $\Phi$ is singular. However, numerical implementation of this inverse Laplace transform is typically ill-conditioned and sensitive to statistical noise, ${ }^{54}$ which is unavoidable in the simulation-based thermal rate calculations.

To ameliorate this problem, we first employ the maximum entropy (MaxEnt) method, ${ }^{55,56}$ which utilizes statistic inference and a Bayesian prior to regularize the numerical inversion. ${ }^{30,56-59}$ MaxEnt is implemented by rewriting the integral in Eq. (7) in matrix form,

$$
\kappa=\mathrm{B} \boldsymbol{v}
$$

where $\boldsymbol{\kappa}$ is the vector of thermal rate input data at discrete temperature points $\left\{\beta_{i}\right\}$, and $v$ is the vector of microcanonical rate outputs at discrete energy values $\left\{E_{j}\right\}$. Specifically, the elements of $\boldsymbol{\kappa}$ and $\boldsymbol{v}$ are $\kappa_{i}=2 \pi \hbar k\left(\beta_{i}\right) Q_{r}\left(\beta_{i}\right)$ and $v_{j}=N\left(E_{j}\right)$. The matrix $\mathbf{B}$ is comprised 
of the Boltzmann kernel $B_{i j}=e^{-\beta_{i} E_{j}} \Delta E_{j}$, where $\Delta E_{j}=E_{j+1}-E_{j}$ is the integration stepsize. MaxEnt yields the microcanonical rate by maximizing the objective function

$$
Q(\boldsymbol{v} ; \alpha)=\alpha S(\boldsymbol{v})-\chi^{2}(\boldsymbol{v}) / 2+V_{\mathrm{reg}}(\boldsymbol{v}),
$$

where the information entropy $S$ describes the degree to which the solution is faithful to a prior model $\lambda\left(\left\{E_{j}\right\}\right)$,

$$
S(v)=\sum_{j}\left(v_{j}-\lambda_{j}-v_{j} \log \frac{v_{j}}{\lambda_{j}}\right),
$$

and the likelihood function $\chi^{2}$ describes the accuracy with which the reference thermal rate data are fit,

$$
\chi^{2}(\boldsymbol{v})=(\boldsymbol{\kappa}-\mathbf{B} \boldsymbol{v})^{\mathrm{T}} \mathbf{C}^{-1}(\boldsymbol{\kappa}-\mathbf{B} \boldsymbol{v}) .
$$

Here, $\mathbf{C}$ is the covariance matrix for the thermal rate data with elements

$$
C_{i i^{\prime}}=\delta_{i i^{\prime}} \sigma_{i}^{2},
$$

where $\delta_{i i^{\prime}}$ is the Kronecker delta function, and $\sigma_{i}$ is the standard deviation for the $i$ th thermal rate datapoint. The parameter $\alpha$ balances between accurately fitting the reference data while preserving the prior model.

Finally, $V_{\text {reg }}(\boldsymbol{v})$ penalizes those trial solutions that violate the physical constraints of the microcanonical rate constant, namely, that it satisfies $N(E) \in[0,1]$. The lower bound is enforced by conducting a solution search only in the positive subspace, while the upper bound is enforced via the functional form

$$
V_{\text {reg }}(\boldsymbol{v})=-\sum_{j} \frac{1}{2} \zeta I^{2}\left(v_{j}-1\right)
$$

where

$$
I\left(v_{j}-1\right)= \begin{cases}v_{j}-1, & v_{j} \geq 1, \\ 0, & v_{j}<1,\end{cases}
$$

and $\zeta$ is chosen according to a tolerance criterion.

\section{Stationary phase approximation}

As an alternative to numerically exact inversion, we apply the stationary phase approximation (SPA) ${ }^{60}$ to Eq. (8). Implementation of the SPA involves finding the stationary point of the phase function $\Phi$ in Eq. (9) and then approximating the integrand as a Gaussian function along the imaginary axis. Setting the first-order derivative of the phase function to zero yields the energy-temperature correspondence $^{61,62}$

$$
E_{\mathrm{st}}=-\left.\frac{\partial \log \left[k(\beta) Q_{r}(\beta)\right]}{\partial \beta}\right|_{\beta_{\mathrm{st}}}, \quad \beta_{\mathrm{st}} \in \mathbb{R},
$$

where $\beta_{\text {st }}$ is the stationary temperature that is assumed to dominate the integrand. The resulting SPA microcanonical rate prediction is given by

$$
N_{\mathrm{SPA}}\left(E_{\mathrm{st}}\right)=\frac{2 \pi \hbar}{\sqrt{2 \pi}}\left(\left.\frac{\partial^{2} \log \left[k(\beta) Q_{r}(\beta)\right]}{\partial \beta^{2}}\right|_{\beta_{\mathrm{st}}}\right)^{-1 / 2} e^{\beta_{\mathrm{st}} E_{\mathrm{st}}} k\left(\beta_{\mathrm{st}}\right) Q_{r}\left(\beta_{\mathrm{st}}\right) .
$$

We note that semiclassical instanton theory can also yield approximate microcanonical rates via the SPA. ${ }^{63}$

A well-known shortcoming of the SPA is that the calculated microcanonical rate violates the constraint $N(E) \leq 1$ in the highenergy limit. ${ }^{62}$ In this regime, the barrier-crossing dynamics reduces to free particle motion, and the thermal rate becomes

$$
\left[k Q_{r}\right]^{\mathrm{FP}}=1 /(2 \pi \beta \hbar) .
$$

Substituting Eq. (19) into Eqs. (17) and (18) yields the energytemperature correspondence relation

$$
E_{\mathrm{st}}^{\mathrm{FP}}=1 / \beta_{\mathrm{st}}^{\mathrm{FP}},
$$

and the corresponding microcanonical rate in the high-energy limit is

$$
N_{\mathrm{SPA}}^{\mathrm{FP}}(E)=e / \sqrt{2 \pi} \simeq 1.084
$$

in excess of the correct upper limit.

\section{Direct shooting approximation}

By analogy with classical rate theory, a physically intuitive strategy for approximating microcanonical rates from RPMD is to simply (i) initialize trajectories from the reactant side with specified translational energy, (ii) propagate those trajectories using the microcanonical equations of motion in Eq. (5), and (iii) count the proportion of trajectories that reach the product region, such that

$$
\begin{aligned}
N_{\text {direct }}\left(E, \beta_{\text {int }}\right)= & \lim _{n \rightarrow \infty} \lim _{t \rightarrow{ }^{\prime \prime} \infty} \frac{n^{n}}{(2 \pi \hbar)^{n-1}} \int d \mathbf{p}_{0} d \mathbf{q}_{0} \frac{e^{-\beta_{\text {int }} H_{n}^{\text {iso }}\left(\mathbf{p}_{0}, \mathbf{q}_{0}\right)}}{e^{-\beta_{\text {int }} E}} \\
& \times \delta\left[\bar{p}_{0}-\sqrt{2 m\left(E-V_{a}\right)}\right] \delta\left(\bar{q}_{0}-q^{\ddagger}\right) h\left(\bar{q}_{t}-q^{\ddagger}\right),
\end{aligned}
$$

where $\bar{p}=\sum_{\alpha} p_{\alpha}$ is the centroid momentum, and $V_{a}$ is the potential energy in the reactant asymptotic region. The centroid kinetic energy for the RPMD trajectories are initialized to match the physical incident energy (as indicated by the $\delta$ function), while the internal modes are thermally sampled from an internal temperature $\beta_{\text {int }}$, the appropriate value of which is not obvious. Previously, ${ }^{44}$ the direct shooting method for calculating microcanonical rates has been applied with the internal temperature set in correspondence to the physical incident energy $\beta_{\text {int }}=1 / E$, which we call the freeparticle protocol; in this work, we shall also consider a prescription for the internal temperature that is derived from the SPA. We note that direct shooting is similar in practical implementation to the calculation of non-equilibrium time-correlation functions using RPMD with momentum-kick initial conditions, ${ }^{9,43}$ although the theoretical justification is more clearly established for the case of nonequilibrium time-correlation functions than for the calculation of microcanonical rates as considered here.

\section{COMPUTATIONAL DETAILS}

Unless specified, all results are reported in atomic units.

Implementation of the direct-shooting approach for microcanonical rates employs Eq. (22). Initial configurations for the ring 
polymer are sampled from the thermal distribution associated with the specified internal temperature $\left(\beta_{\text {int }}\right)$, while dynamical evolution is performed using the standard RPMD integration scheme with a time step of 0.3 . This choice of time step is confirmed to avoid resonance instabilities, although we note that the best practice for future applications is to employ the Cayley-modification to the RPMD integration. ${ }^{6,67}$ Calculations with up to 144 ring-polymer beads are performed to ensure the convergence of the path-integral discretization.

Implementation of the SPA utilizes Eqs. (17) and (18). Equation (17) is first solved to obtain the stationary temperature from the thermal rate data. Then, the SPA microcanonical rate is obtained using Eq. (18). First- and second-order derivatives of $k Q_{r}$ are obtained from a standard basis-spline interpolation procedure. ${ }^{68,69}$ Validation of the numerical procedure is performed by comparison with independent SPA results obtained from path-integral Monte Carlo sampling methods (see the Appendix).

Implementation of the MaxEnt approach closely follows the Bryan algorithm. ${ }^{55,56}$ Calculations are performed with a modified version of an open-source code. ${ }^{70}$ Quantum mechanical and RPMD thermal rates are the fitting targets in these calculations, while SPA microcanonical rates are employed as the Bayesian prior. For the exact quantum mechanical rate inversion, the diagonal elements of the covariance matrix in Eq. (14) are set to $0.4 \%$ of the rate value, as is consistent with the statistical noise in the RPMD thermal rate calculations. Although not included here, MaxEnt results were also obtained using the flat Bayesian prior, $\lambda(E)=1$; however, the numerics of these calculations were found to be less stable than those based on the SPA, which requires no additional information beyond the thermal rates that are also used for the fitting target. To ensure that the SPA priors are nonzero and sufficiently smooth, they are filtered with a low threshold value of $10^{-3}$, followed by a simple moving average procedure to suppress the statistical fluctuations. A regulation potential [Eq. (15)] with $\zeta=10^{7}$ is sufficient to enforce the upper bound of $N(E)$ to a tolerance of tight $10^{-4}$ in all reported calculations.

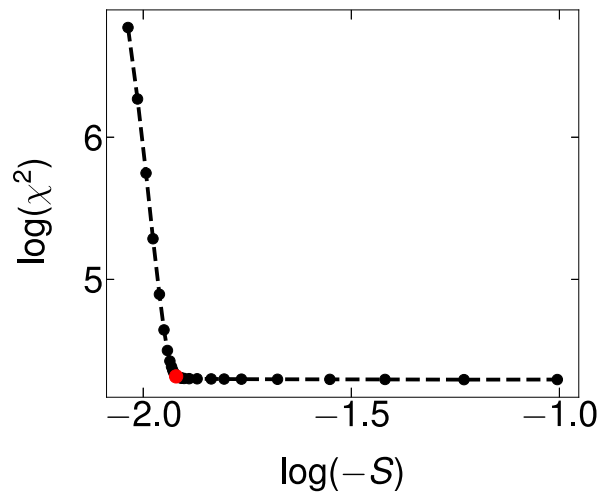

FIG. 1. An illustrative example of the "L-curve" that is used to determine the parameter $\alpha$ in each MaxEnt calculation. The optimal value of $\alpha$ coincides with the kink in the curve (indicated by a red point). This example corresponds to the Eckart barrier, using RPMD thermal rate data for the fitting target and the SPA-RPMD microcanonical rates for the Bayesian prior.
To specify the parameter $\alpha$ in the objective function of the MaxEnt calculations [Eq. (11)], the "L-curve" rule was employed as in many previous studies. ${ }^{30,57,58}$ As illustrated with a representative example in Fig. 1, the balance between fitting accuracy and solution likelihood when plotted as a parametric function of $\alpha$ yields a hockey stick-shaped curve. We take the kink of the curve (red point) to correspond to the optimal balance between these attributes.

\section{RESULTS}

\section{A. Microcanonical RPMD rates for electronically adiabatic reactions}

We begin by analyzing the effectiveness of the direct shooting approach with different choices of ring-polymer internal temperatures, $\beta_{\text {int }}$. The symmetric Eckart barrier model for $\mathrm{H}+\mathrm{H}_{2}$ reactive scattering ${ }^{4}$ is chosen as the test example, with a potential energy function

$$
V(q)=V_{0} / \cosh ^{2}\left(q / q_{0}\right)
$$

using parameters $m=1061, V_{0}=0.425 \mathrm{eV}$, and $q_{0}=0.734$. The analytical solution of the microcanonical rate for this model yields

$$
N(E)=f /(f+g)
$$

where

$$
f=\sinh ^{2}\left(\pi q_{0} \sqrt{2 m E} / \hbar\right)
$$

and

$$
g=\cosh ^{2}\left(\pi \sqrt{\left|2 m V_{0} q_{0}^{2} / \hbar^{2}-1 / 4\right|}\right),
$$

and the exact thermal rate is obtained by integrating $N(E)$ over the Boltzmann kernel following Eq. (7).

\section{Free-particle direct shooting}

Figure 2(a) plots the microcanonical rate prediction from classical mechanics, exact quantum mechanics, and the direct shooting scheme [Eq. (22)], as a function of energy. As expected, the stepfunction shape of the classical result is smoothed due to nuclear quantum effects. It is clear from the figure that the direct-shooting scheme is strongly sensitive to the choice of internal ring-polymer temperature, particularly at low energies for which tunneling plays an important role; irrespective of the employed value of $\beta_{\text {int }}$, the direct-shooting scheme reverts to classical behavior in the highenergy regime. Strikingly, almost all nuclear quantum effects are absent using the free-particle protocol $\left(\beta_{\text {int }}=1 / E\right)$ for the internal ring-polymer temperature.

Figure 2(b) presents the canonical reaction rates for the Eckart barrier as a function of temperature, including exact quantum and classical results, as well as the standard RPMD calculation of the thermal reaction rate (green line). ${ }^{3,4}$ As is well known for such problems, RPMD allows for the direct calculation of thermal reaction rates with good accuracy; it is exact at high temperatures ${ }^{4}$ and works well at low temperatures due to its connection to 

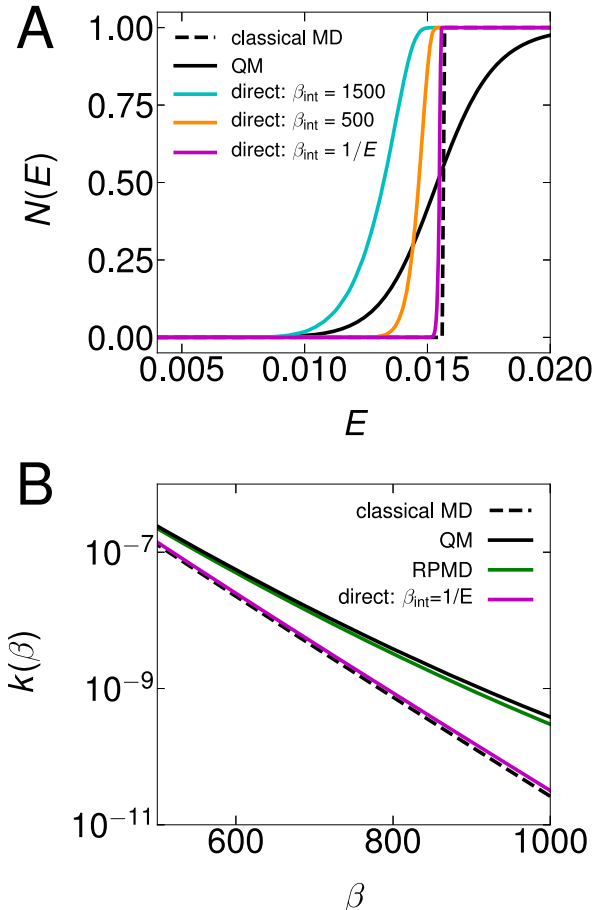

FIG. 2. (a) Microcanonical rate predictions for the Eckart barrier. Results are calculated with classical mechanics (classical MD, dashed black line), analytical quantum mechanics (QM, solid black line), and direct shooting approach with different internal ring-polymer temperatures (direct: solid cyan, orange, and magenta lines). The free-particle direct shooting protocol is labeled $\beta_{\text {int }}=1 / E$. (b) Thermal rates obtained by substituting microcanonical rates from various levels of theory (classical MD, QM, and free-particle direct shooting) into Eq. (7). For comparison, the standard RPMD thermal rates (green line) are also included.

semiclassical instanton theory. ${ }^{71}$ However, the figure also shows the results of the RPMD thermal rate prediction obtained by transforming [via Eq. (7)] the microcanonical RPMD rates from the free-particle direct-shooting protocol (magenta line). Consistent with the results of Fig. 2(a), this direct-shooting protocol provides an essentially classical description of the thermal reaction rate across the entire range of temperatures. Figure 2(b) clearly demonstrates that approximation of microcanonical rates via the free-particle direct-shooting method (magenta vs solid black line) is a far greater source of error than the intrinsic approximation of RPMD for calculating thermal rates (green vs solid black line). This figure illustrates the hazards of using direct shooting for RPMD microcanonical rates, and it suggests that better results for microcanonical rate should be achievable on the basis of RPMD dynamics.

\section{Stationary phase approximation}

We now turn our attention to the use of the SPA to calculate RPMD microcanonical reaction rates for the example of the Eckart barrier. Figure 3(a) presents the calculated stationary temperature $\beta_{\text {st }}$ as a function of energy obtained via Eq. (17) with input
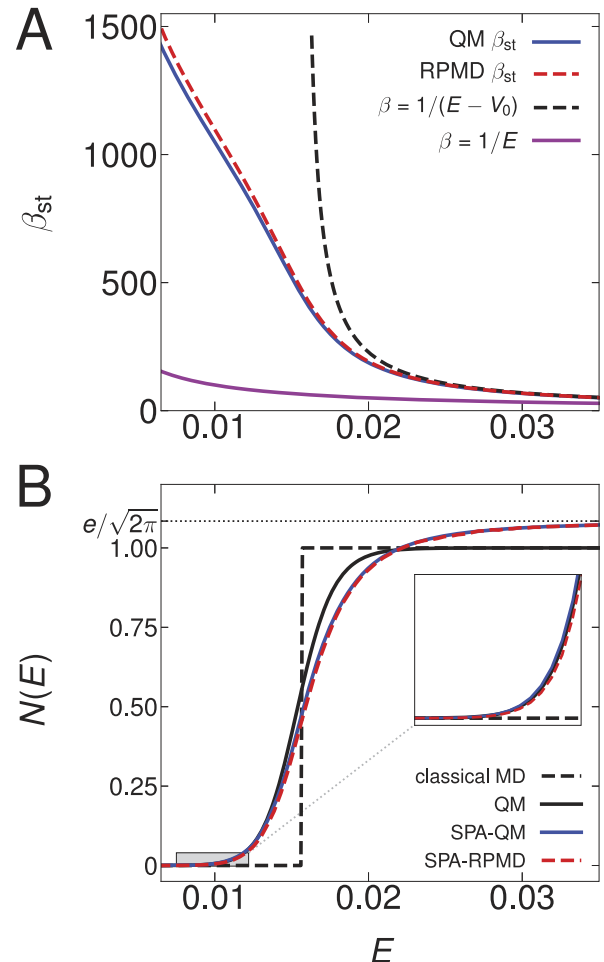

FIG. 3. Stationary phase approximation (SPA) results for the Eckart barrier. (a) The stationary temperature calculated with QM (blue line) and RPMD (dashed red line) thermal rates, respectively, as a function of incident energy. For comparison, the classical asymptote of the stationary temperature (dashed black line) and the free-particle temperature (magenta line) are also included. (b) Microcanonical rates obtained using the SPA with input from QM (blue line) and RPMD (dashed red line) thermal rates. For comparison, the microcanonical rates from exact QM and classical MD are also included. The inset expands the low-energy regime.

from either exact quantum thermal rates (blue line) or standard RPMD thermal rate calculations (dashed red line). For comparison, we also show the temperature associated with the free-particle protocol $(\beta=1 / E)$, which differs significantly from the stationary temperature at each energy, as well as an analytical expression for the high-energy stationary temperature $\left[\beta=1 /\left(E-V_{0}\right)\right]$ that is derived in the Appendix. In the low-energy regime, only the RPMD thermal rate data provides a satisfactory description of the stationary temperature obtained from the exact quantum results.

Figure 3(b) presents microcanonical rates from the SPA [Eq. (18)] using input from RPMD thermal rates. To provide a baseline of accuracy associated with the SPA, we first compare the microcanonical rate from exact quantum mechanics (solid black line) with that obtained via SPA applied to exact quantum thermal rates (blue line). The difference in these curves presents a best-case scenario for the accuracy of a method that approximates microcanonical rates via SPA; moreover, it is seen that while the agreement at low energy is excellent, there is substantial deviation associated with energies in the high-energy regime. Encouragingly, essentially identical performance is seen when the microcanonical rates are 
obtained via application of the SPA to RPMD thermal rates (dashed red line). This indicates that the RPMD thermal rates are a smaller source of error than the SPA in the high-energy regime. Finally, a comparison of the results in Fig. 3(b) with Fig. 2(a) makes clear that RPMD offers a much more accurate avenue to the calculation of microcanonical rates than might be concluded from simulations that employ direct shooting.

We again note that the SPA errors at high energy in Fig. 3(b) that are well known and due to the neglect of higher-order terms in the phase function. ${ }^{62}$ As anticipated from Eq. (21), both sets of SPA results in the figure converge to the erroneous high-energy asymptote of 1.084 .

\section{Maximum entropy inversion}

As the third alternative for obtaining microcanonical rates from RPMD, Fig. 4(a) presents results for the Eckart barrier obtained using MaxEnt inversion. To establish the baseline error for the MaxEnt procedure, the dashed blue curve presents the results obtained via inversion of the exact quantum mechanics (QM) thermal rates using the SPA-QM microcanonical rates [Fig. 3(b)] as the Bayesian prior. In addition, the dashed red curve presents the MaxEnt results obtained via inversion of the RPMD thermal rates using the SPARPMD microcanonical rates as the Bayesian prior. This last result utilizes input from RPMD thermal rates alone.

It is clear from Fig. 4(a) that the MaxEnt procedure provides excellent accuracy across the entire range of energies on the scale plotted, avoiding the incorrect high-energy asymptote of the SPA results. A closer examination of the low-energy regime in the inset shows that the agreement persists even in the regime of strong tunneling. The comparison of the SPA results (SPA-QM and SPARPMD) with their MaxEnt refinements, in Fig. 4(b), reveals that MaxEnt can introduce significant relative errors in the rate predictions at low energies for which the rates are very small. Taken together, the results in Figs. 4(a) and 4(b) indicate that for this example, the use of MaxEnt inversion helps improve the quality of the SPA at intermediate and higher energies, but it does little to improve the quality of the SPA in the low-energy regime.

Finally, as a self-consistency check, Fig. 4(c) presents the thermal rates obtained by transforming [via Eq. (7)] the microcanonical rates obtained from the MaxEnt inversion of the RPMD thermal rates (dashed red line). For comparison, the exact quantum, classical, and standard RPMD thermal rates are also included. As expected, the MaxEnt RPMD rates are fully consistent with the standard RPMD thermal rates, and both are in good agreement with the exact QM results.

\section{Stationary-temperature direct shooting}

Given the success of the SPA for extracting microcanonical rates from standard RPMD thermal rates, it is tempting to see whether data obtained from the SPA can be used to improve the direct shooting method. Specifically, we explore the use of the stationary temperature as the ring-polymer internal temperature for initializing and propagating the direct-shooting trajectories, i.e., setting $\beta_{\text {int }}=\beta_{\text {st }}$ in Eq. (22). This strategy is physically appealing, since the stationary temperature [which is a function of incident energy; see Fig. 3(a)] dictates the delocalization of the ring-polymer in its barrier-crossing configuration; ${ }^{61,63,71}$ moreover, note that the
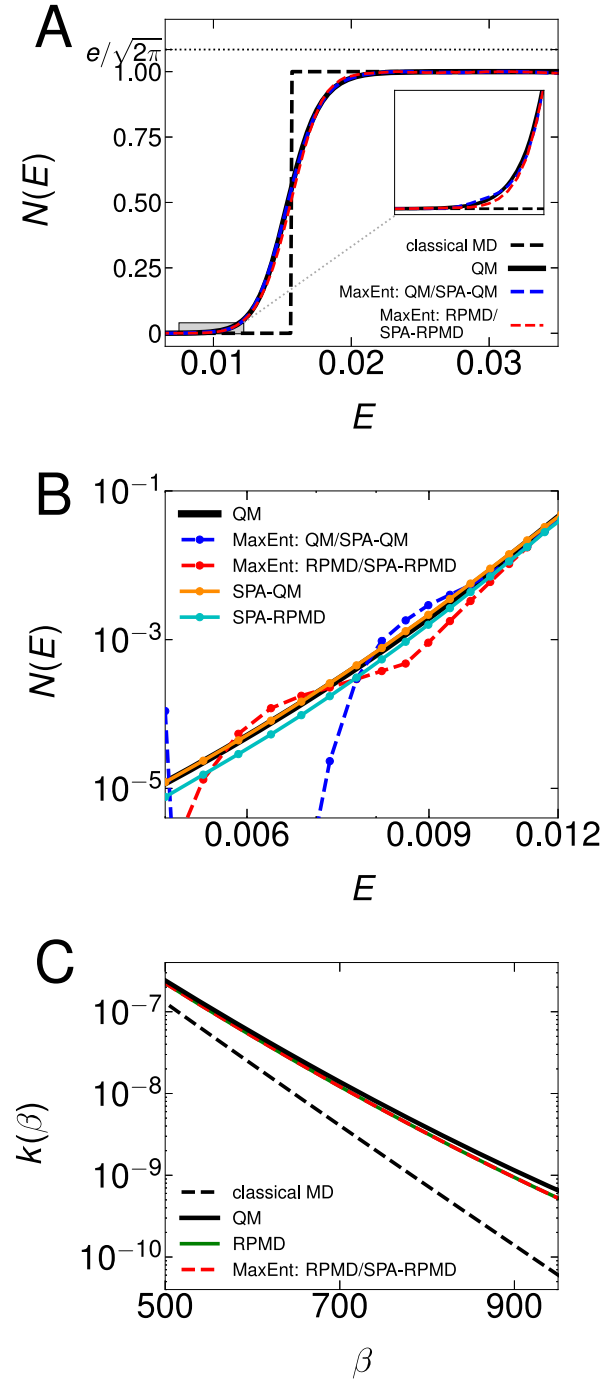

FIG. 4. Maximum entropy (MaxEnt) inversion results for the Eckart barrier. The methods are labeled with the format "MaxEnt-[thermal rate input type]/[prior type]." (a) MaxEnt solutions for the microcanonical reaction rate as a function of incident energy, with the inset expanding the low-energy regime. Microcanonical rates from classical MD and exact QM are also presented for reference. (b) An expanded view of panel (a) in the low-energy regime, plotted on a log-log scale. (c) Thermal rates obtained by integrating $N(E)$ in panel (a) over the Boltzmann kernel. For comparison, exact $\mathrm{QM}$, classical MD and standard RPMD thermal rates are also included.

stationary temperature approaches the free-particle temperature at high incident energies.

Figure 5 plots the microcanonical rate for the Eckart barrier, obtained using the stationary-temperature direct-shooting method (red line). For comparison, the free-particle direct-shooting (magenta line), classical MD (dashed black line), and exact quantum (solid black line) results are all reproduced from Fig. 2(a). While stationary-temperature direct shooting remains qualitatively less accurate than the SPA and MaxEnt inversion methods, it, 


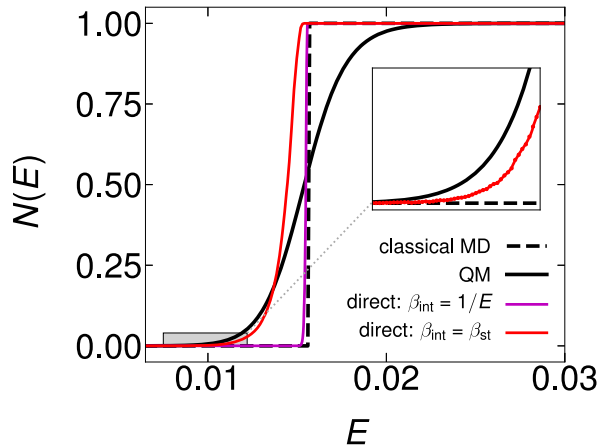

FIG. 5. Microcanonical rate predictions for the Eckart barrier, comparing the directshooting method with the ring-polymer internal temperature set to either the stationary temperature (red line) or the free-particle temperature (magenta line). Also included are the exact QM and classical MD results.

nonetheless, substantially improves the results of the free-particle direct-shooting approach in the low-energy region where tunneling is important. These results indicate that stationary-temperature direct shooting is a less quantitative tool than SPA or MaxEnt for the calculation of microcanonical rates from RPMD trajectories, but it may, nonetheless, prove useful in exploratory studies for which a direct trajectory-based simulation approach is needed or in applications to high-dimensional systems for which obtaining precise thermal reaction rate in the whole temperature region is computationally expensive.

\section{B. Microcanonical RPMD rates for non-adiabatic systems}

Although we have thus far only discussed the SPA and MaxEnt inversion methods in the context of single-level (i.e., electronically adiabatic) processes, both methods can be naturally extended to multi-level systems. Specifically, given state-resolved thermal reaction rates for a non-adiabatic process, both the SPA and MaxEnt methods can be used to compute state-resolved microcanonical rates for different reaction channels.

For the SPA method, state-resolved thermal reaction rates are substituted into Eqs. (17) and (18), yielding a single stationary temperature and a single state-resolved microcanonical rate for each reaction channel, respectively. For the MaxEnt method, we solve the coupled integral equation

$$
\left(\begin{array}{l}
\boldsymbol{\kappa}_{1 \rightarrow 2} \\
\boldsymbol{\kappa}_{2 \rightarrow 2}
\end{array}\right)=\left(\begin{array}{c}
\text { B } \\
\text { B }
\end{array}\right) \cdot\left(\begin{array}{l}
\boldsymbol{v}_{1 \rightarrow 2} \\
\boldsymbol{v}_{2 \rightarrow 2}
\end{array}\right)
$$

for a system with two reaction channels (e.g., diabat $1 \rightarrow 2$ and diabat $2 \rightarrow 2$ ), with $\boldsymbol{\kappa}, \mathbf{B}$, and $\boldsymbol{v}$ defined in Eq. (10). The MaxEnt objective function for the two-level system is

$$
\begin{aligned}
Q\left(\boldsymbol{v}_{1 \rightarrow 2}, \boldsymbol{v}_{2 \rightarrow 2} ; \alpha\right)= & \alpha S\left(\boldsymbol{v}_{1 \rightarrow 2}\right)-\chi^{2}\left(\boldsymbol{v}_{1 \rightarrow 2}\right) / 2 \\
& +\alpha S\left(\boldsymbol{v}_{2 \rightarrow 2}\right)-\chi^{2}\left(\boldsymbol{v}_{2 \rightarrow 2}\right) / 2 \\
& +V_{\text {reg }}\left(\boldsymbol{v}_{1 \rightarrow 2}, \boldsymbol{v}_{2 \rightarrow 2}\right),
\end{aligned}
$$

which sums the information entropy and likelihood function contributions for the state-resolved rates. The regularization potential is likewise generalized,

$$
V_{\text {reg }}(\boldsymbol{v})=-\sum_{j} \frac{1}{2} \zeta I^{2}\left(v_{1 \rightarrow 2, j}+v_{2 \rightarrow 2, j}-1\right),
$$

to enforce unitarity (with the detailed balance condition)

$$
N_{1 \rightarrow 2}\left(E_{j}\right)+N_{2 \rightarrow 2}\left(E_{j}\right) \leq 1 \quad \forall j .
$$

The constraint of non-negativity

$$
N_{1 \rightarrow 2}\left(E_{j}\right) \geq 0, N_{2 \rightarrow 2}\left(E_{j}\right) \geq 0, \quad \forall j
$$

is enforced as before by confining the solution search to the positive subspace.

As a numerical demonstration for non-adiabatic reaction dynamics, we use a two-state gas-phase reactive scattering model that has been previously introduced. ${ }^{41,72}$ In the diabatic representation, the potential energy functions for this system are

$$
\begin{aligned}
& V_{11}(q)=\frac{A_{1}}{1+e^{-a_{1}\left(q-q_{1}\right)}+B_{1},} \\
& V_{22}(q)=\frac{A_{2}}{1+e^{-a_{2} q}}+\frac{B_{2}}{4 \cosh ^{2}\left(a_{2} q / 2\right)}, \\
& V_{12}(q)=V_{21}(q)=A_{3} e^{-a_{3}\left(q-q_{3}\right)^{2}},
\end{aligned}
$$

with parameters specified in Table I and with reactant and product regions corresponding to $q \rightarrow-\infty$ and $q \rightarrow \infty$, respectively.

We focus on microcanonical rates in the range of incident energies for which the higher-energy state is unavailable as a product channel (i.e., the only two available reactions channels correspond to the $1 \rightarrow 2$ and $2 \rightarrow 2$ processes on the diabatic states). Calculation of state-resolved thermal rates for the two channels is performed with the flux-side formulation ${ }^{72}$ of iso-RPSH, ${ }^{41}$ with both the methodological details and thermal-rate results reported elsewhere. ${ }^{72}$ The only difference in the current work is that 1000 -fold more trajectories are performed to suppress statistical error in the thermal rates for the MaxEnt calculations, although the thermal rate results are graphically indistinguishable from those previously published.

For comparison, Fig. 6(a) presents the state-resolved microcanonical rates obtained from numerically exact quantum mechanics $^{73,74}$ and using classical surface hopping ${ }^{75}$ as implemented in our previous work. ${ }^{41}$ As for the one-level system, the microcanonical rates with classical nuclear dynamics exhibit a sharp increase

TABLE I. Parameters for the two-level model in Eq. (30).

\begin{tabular}{lccc}
\hline \hline Parameter & Value & Parameter & Value \\
\hline$A_{1}$ & 7 & $a_{1}$ & 1 \\
$A_{2}$ & $-18 / \pi$ & $a_{2}$ & $\sqrt{3 \pi} / 4$ \\
$A_{3}$ & 0.25 & $a_{3}$ & 0.25 \\
$B_{1}$ & -0.75 & $q_{1}$ & -1.6 \\
$B_{2}$ & $54 / \pi$ & $q_{3}$ & -2.625 \\
\hline \hline
\end{tabular}


when the incident energy reaches the barrier height. Although classical surface hopping qualitatively includes the effect of the nonadiabatic transition and performs well in the high-energy regime, it fails to capture the significant nuclear quantum effects in this problem.

Figure 6(b) presents the microcanonical rates obtained via application of the SPA to state-resolved thermal rates from exact QM (blue line) and iso-RPSH (red line). A comparison of the QM and SPA-QM results indicate that the SPA is a good approximation in this example. Furthermore, a comparison of these curves with the SPA-iso-RPSH results indicates that the iso-RPSH thermal rate data are an even smaller source of error than the SPA. At higher energies,
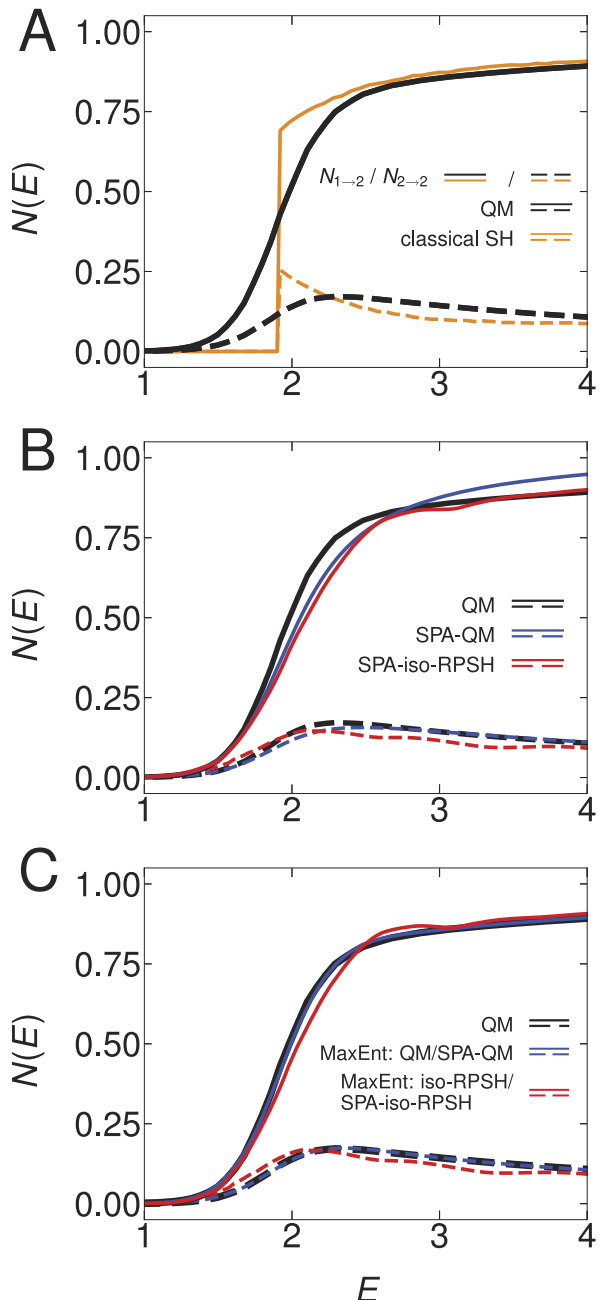

FIG. 6. State-resolved microcanonical rates for the two-level model in Eq. (30). Dashed lines indicate the $1 \rightarrow 2$ diabatic reaction channel and solid lines indicate the $2 \rightarrow 2$ diabatic reaction channel. (a) Microcanonical rates from classical surface hopping (orange line) and numerically exact QM wavepacket propagation (black line). (b) SPA results for the microcanonical rate, with input from exact QM (blue line) and iso-RPSH (red line) thermal rates. (c) MaxEnt results for the microcanonical rate, with input from exact QM (blue line) and iso-RPSH (red line) thermal rates. the SPA results exhibit the same pathologies as those discussed in connection with Eq. (21), and the better performance of SPA-isoRPSH vs SPA-QM in this regime is likely due to fortuitous error cancellation.

Figure 6(c) presents the microcanonical rates obtained via application of the MaxEnt method to the state-resolved thermal rates. As for the one-level system described in Fig. 4, the MaxEnt method improves the description for the two-level system at high energies but does little to refine the description of the SPA at lower energies.

Taken together, these results indicate that the iso-RPSH method can be straightforwardly extended for the accurate calculation of state-resolved microcanonical rates. Moreover, these results show that iso-RPSH provides an accurate description of both the thermal and microcanonical reaction rates of this system in a regime for which both non-adiabatic and nuclear quantum effects play an important role, although the method has been shown to underestimate the asymmetry in the Marcus inverted regime in the golden-rule limit of electron transfer. ${ }^{7}$

\section{SUMMARY}

Although the ring-polymer molecular dynamics (RPMD) thermal rate theory has proven immensely successful in many chemical application domains, far less attention has been paid to the problem of calculating microcanonical reaction rates using RPMD, which may be of considerable value in the context of gas-phase and surface-molecule scattering processes for which nuclear quantization plays an important role. ${ }^{77-79}$ The current work addresses this shortcoming by exploring a variety of strategies to calculate microcanonical reaction rates with RPMD. It is found that the ad hoc strategy of direct shooting of RPMD trajectories is strongly sensitive to the internal ring-polymer temperature that is employed; this is somewhat ameliorated in the tunneling regime via the use of an internal temperature based on the stationary-phase approximation (SPA), but the resulting direct-shooting results remain overly classical in the barrier-crossing energy regime. Far more accurate microcanonical rates are obtained from RPMD thermal rate data via Laplace transform inversion using either the SPA or the numerically exact maximum entropy method. In general, we find that the SPA applied to RPMD thermal rate data provides the best compromise between good accuracy and numerical feasibility, particularly in the low-energy tunneling regime, although we point out that the alternative direct-shooting and maximum entropy methods described here may also prove useful in particular application cases.

Although this paper focuses only on the calculation of microcanonical rates from RPMD (or other approximate) thermal rate data, we note that the same strategy can be applied for the calculation of collisional cross section and other time correlation functions, spectra, and transport coefficients in the microcanonical ensemble.

\section{ACKNOWLEDGMENTS}

We acknowledge support from the Department of Energy under Award No. DE-FOA-0001912 and the National Science Foundation under Award No. CHE-1611581. P.S. acknowledges 
a German Research Foundation (DFG) postdoctoral fellowship. Computational resources were provided by the National Energy Research Scientific Computing Center, which is supported by the Office of Science of the U.S. Department of Energy under Contract No. DE-AC02-05CH11231.

\section{APPENDIX: EVALUATION OF THE STATIONARY TEMPERATURE VIA PATH-INTEGRAL MONTE CARLO SAMPLING METHODS}

As an alternative method for evaluating the stationary temperature $\beta_{\text {st }}$ in Eq. (17), we apply path-integral Monte Carlo sampling methods in combination with quantum transition state theory (QTST) approximation ${ }^{80}$ to the reaction rate $^{81-83}$

$$
k(\beta) \simeq k_{\mathrm{QTST}}\left(\beta, q_{\mathrm{O}}^{\ddagger}\right)=\min _{q^{\ddagger}}\left[k_{\mathrm{QTST}}\left(\beta, q^{\ddagger}\right)\right]
$$

with a dividing surface $q_{\mathrm{o}}^{\ddagger}$ that minimizes dynamical recrossing effects. Using the path-integral representation of the QTST rate in the case of a single-surface system,

$$
\begin{aligned}
k_{\mathrm{QTST}}\left(\beta, q_{\mathrm{o}}^{\ddagger}\right) Q_{r}= & \lim _{n \rightarrow \infty}\left(\frac{n}{2 \pi \hbar}\right)^{n} \int d \mathbf{p}_{0} d \mathbf{q}_{0} e^{-\beta H_{n}^{\mathrm{iso}}(\mathbf{p}, \mathbf{q})} \\
& \times \delta\left(\bar{q}_{0}-q_{\mathrm{o}}^{\ddagger}\right) \bar{v}_{0} h\left(\bar{v}_{0}\right),
\end{aligned}
$$

together with Euler's theorem for homogeneous functions, ${ }^{84}$ we derive a virial-like expression for the stationary energy-temperature relation, which can be conveniently evaluated using path-integral Monte Carlo sampling methods as follows:

$$
E_{\mathrm{st}}=\frac{1}{\beta_{\mathrm{st}}}+\left\langle\frac{1}{2 n} \sum_{\alpha}\left(q_{\alpha}-\bar{q}_{0}\right) \frac{\partial V\left(q_{\alpha}\right)}{\partial q_{\alpha}}+\frac{1}{n} \sum_{\alpha} V\left(q_{\alpha}\right)\right\rangle_{q_{\mathrm{o}}^{\ddagger}} .
$$

Here, $\langle\mathcal{Q}\rangle_{q_{0}^{\ddagger}}$ is a constrained ensemble average defined by

$$
\langle\mathcal{Q}\rangle_{q_{\mathrm{o}}^{\ddagger}}=\frac{\int d \mathbf{q}_{0} \rho_{c}\left(\mathbf{q}_{0}\right) \mathcal{Q}\left(\mathbf{q}_{0}\right)}{\int d \mathbf{q}_{0} \rho_{c}\left(\mathbf{q}_{0}\right)},
$$

where

$$
\rho_{c}\left(\mathbf{q}_{0}\right)=\delta\left(\bar{q}_{0}-q_{\mathrm{o}}^{\ddagger}\right) e^{-\beta U_{\text {spr }}\left(\mathbf{q}_{0}\right)} e^{-\beta_{n} \sum_{\alpha} V\left(q_{\alpha}\right)} .
$$

This approximate method for obtaining $\beta_{\text {st }}$ works well for the numerical examples considered here, although it is more limited than the general numerical differentiation method that is described in Sec. III.

Now, let us proceed by analyzing the high-temperature asymptotic behavior of the stationary temperature. At high temperatures, Eq. (A3) approaches the classical limit for the stationary energytemperature relation

$$
\lim _{\beta_{\mathrm{st}} \rightarrow 0} E_{\mathrm{st}}=\frac{1}{\beta_{\mathrm{st}}}+V\left(q_{\mathrm{o}}^{\ddagger}\right)
$$

with $q_{\mathrm{o}}^{\ddagger}$ approaching the barrier top. This classical limit can also be obtained upon the substitution of the classical transition state theory rate,

$$
\left[k Q_{r}\right]^{\mathrm{CTST}}=\frac{1}{2 \pi \beta \hbar} e^{-\beta V\left(q_{\mathrm{o}}^{\ddagger}\right)},
$$

into Eq. (17), yielding

$$
E_{\text {st }}=-\left.\frac{\partial \log \left[k^{\mathrm{CTST}}(\beta) Q_{r}(\beta)\right]}{\partial \beta}\right|_{\beta_{\mathrm{st}}}=\frac{1}{\beta_{\mathrm{st}}}+V\left(q_{\mathrm{o}}^{\ddagger}\right) .
$$

\section{REFERENCES}

${ }^{1}$ I. R. Craig and D. E. Manolopoulos, J. Chem. Phys. 121, 3368-3373 (2004).

${ }^{2}$ S. Habershon, D. E. Manolopoulos, T. E. Markland, and T. F. Miller III, Annu. Rev. Phys. Chem. 64, 387-413 (2013).

${ }^{3}$ I. R. Craig and D. E. Manolopoulos, J. Chem. Phys. 122, 084106 (2005).

${ }^{4}$ I. R. Craig and D. E. Manolopoulos, J. Chem. Phys. 123, 034102 (2005).

${ }^{\mathbf{5}}$ S. Habershon, G. S. Fanourgakis, and D. E. Manolopoulos, J. Chem. Phys. 129, 074501 (2008).

${ }^{6}$ M. Rossi, M. Ceriotti, and D. E. Manolopoulos, J. Chem. Phys. 140, 234116 (2014).

${ }^{7}$ T. F. Miller III and D. E. Manolopoulos, J. Chem. Phys. 122, 184503 (2005).

${ }^{8}$ T. F. Miller III and D. E. Manolopoulos, J. Chem. Phys. 123, 154504 (2005).

${ }^{9}$ H. Jiang, M. Kammler, F. Ding, Y. Dorenkamp, F. R. Manby, A. M. Wodtke, T. F. Miller, A. Kandratsenka, and O. Bünermann, Science 364, 379-382 (2019).

${ }^{10}$ Q. Liu, L. Zhang, Y. Li, and B. Jiang, J. Phys. Chem. Lett. 10, 7475-7481 (2019).

${ }^{11}$ R. Kaur and R. Welsch, J. Chem. Phys. 150, 114105 (2019).

${ }^{12}$ Q. Yu and J. M. Bowman, J. Phys. Chem. A 123, 1399-1409 (2019).

${ }^{13}$ I. S. Novikov, Y. V. Suleimanov, and A. V. Shapeev, Phys. Chem. Chem. Phys. 20, 29503-29512 (2018).

${ }^{14}$ D. M. Wilkins, D. E. Manolopoulos, S. Pipolo, D. Laage, and J. T. Hynes, J. Phys. Chem. Lett. 8, 2602-2607 (2017).

${ }^{15}$ K. Kreis, K. Kremer, R. Potestio, and M. E. Tuckerman, J. Chem. Phys. 147, 244104 (2017).

${ }^{16}$ M. Rossi, M. Ceriotti, and D. E. Manolopoulos, J. Phys. Chem. Lett. 7, 30013007 (2016).

${ }^{17}$ R. L. Kenion and N. Ananth, Phys. Chem. Chem. Phys. 18, 26117-26124 (2016).

${ }^{18}$ Y. V. Suleimanov, F. J. Aoiz, and H. Guo, J. Phys. Chem. A 120, 8488-8502 (2016).

${ }^{19}$ M. Ceriotti, J. Cuny, M. Parrinello, and D. E. Manolopoulos, Proc. Natl. Acad. Sci. U. S. A. 110, 15591-15596 (2013).

${ }^{20}$ O. Marsalek and T. E. Markland, J. Chem. Phys. 144, 054112 (2016).

${ }^{21}$ Y. Li, Y. V. Suleimanov, J. Li, W. H. Green, and H. Guo, J. Chem. Phys. 138, 094307 (2013).

${ }^{22}$ R. Pérez de Tudela, F. J. Aoiz, Y. V. Suleimanov, and D. E. Manolopoulos, J. Phys. Chem. Lett. 3, 493-497 (2012).

${ }^{23}$ Y. V. Suleimanov, J. Phys. Chem. C 116, 11141-11153 (2012).

${ }^{24}$ J. S. Kretchmer and T. F. Miller III, Inorg. Chem. 55, 1022-1031 (2015).

${ }^{25}$ J. S. Kretchmer and T. F. Miller III, J. Chem. Phys. 138, 134109 (2013).

${ }^{26}$ A. R. Menzeleev, N. Ananth, and T. F. Miller III, J. Chem. Phys. 135, 074106 (2011).

${ }^{27}$ A. R. Menzeleev and T. F. Miller III, J. Chem. Phys. 132, 034106 (2010).

${ }^{28}$ R. Collepardo-Guevara, Y. V. Suleimanov, and D. E. Manolopoulos, J. Chem. Phys. 130, 174713 (2009).

${ }^{29}$ N. Boekelheide, R. Salomon-Ferrer, and T. F. Miller, Proc. Natl. Acad. Sci. U. S. A. 108, 16159-16163 (2011).

${ }^{30}$ T. F. Miller III, J. Chem. Phys. 129, 194502 (2008).

${ }^{31}$ T. J. H. Hele, M.Sc. thesis, Exeter College, Oxford University, 2011.

${ }^{32}$ J. R. Duke and N. Ananth, Faraday Discuss. 195, 253-268 (2017).

${ }^{33}$ A. R. Menzeleev, F. Bell, and T. F. Miller III, J. Chem. Phys. 140, 064103 (2014).

${ }^{34}$ J. S. Kretchmer and T. F. Miller III, Faraday Discuss. 195, 191-214 (2017). 
${ }^{35}$ J. S. Kretchmer, N. Boekelheide, J. J. Warren, J. R. Winkler, H. B. Gray, and T. F. Miller III, Proc. Natl. Acad. Sci. U. S. A. 115, 6129-6134 (2018).

${ }^{36}$ N. Ananth, J. Chem. Phys. 139, 124102 (2013).

${ }^{37}$ S. Pierre, J. R. Duke, T. J. H. Hele, and N. Ananth, J. Chem. Phys. 147, 234103 (2017).

${ }^{38}$ J. O. Richardson and M. Thoss, J. Chem. Phys. 139, 031102 (2013).

${ }^{39}$ S. N. Chowdhury and P. Huo, J. Chem. Phys. 147, 214109 (2017).

${ }^{40}$ S. N. Chowdhury and P. Huo, J. Chem. Phys. 150, 244102 (2019).

${ }^{41}$ X. Tao, P. Shushkov, and T. F. Miller III, J. Chem. Phys. 148, 102327 (2018).

${ }^{42}$ P. Shushkov, R. Li, and J. C. Tully, J. Chem. Phys. 137, 22 A549 (2012).

${ }^{43}$ R. Welsch, K. Song, Q. Shi, S. C. Althorpe, and T. F. Miller III, J. Chem. Phys. 145, 204118 (2016).

${ }^{44}$ J. R. Duke and N. Ananth, J. Phys. Chem. Lett. 6, 4219-4223 (2015).

${ }^{45}$ F. A. Shakib and P. Huo, J. Phys. Chem. Lett. 8, 3073-3080 (2017).

${ }^{46}$ R. P. Feynman and A. R. Hibbs, Quantum Mechanics and Path Integrals (McGraw-Hill, 1965).

${ }^{47}$ D. Chandler and P. G. Wolynes, J. Chem. Phys. 74, 4078-4095 (1981).

${ }^{48} \mathrm{M}$. Parrinello and A. Rahman, J. Chem. Phys. 80, 860-867 (1984).

${ }^{49}$ Following previous work, ${ }^{50}$ we refer to $N(E)$ as the microcanonical rate, although it is also known as the cumulative reaction probability. The quantity $r(E)=N(E) /[2 \pi \hbar \rho(E)]$ is also commonly referred to as the microcanonical rate, where $\rho(E)$ is the density of states; by estimating $\rho(E)$ with statistical mechanical methods, the quantities can be interconverted.

${ }^{50}$ W. H. Miller, J. Chem. Phys. 63, 1166-1172 (1975).

${ }^{51}$ N. Slater, Proc. Leeds Philos. Lit. Soc., Sci. Sect. 6, 259 (1955).

${ }^{52}$ J. W. Davies, N. J. B. Green, and M. J. Pilling, Chem. Phys. Lett. 126, 373-379 (1986).

${ }^{53}$ D. S. Firaha, M. Döntgen, B. Berkels, and K. Leonhard, ACS Omega 3, 82128219 (2018).

${ }^{54}$ W. H. Press, Numerical Recipes, The Art of Scientific Computing, 3rd ed. (Cambridge University Press, 2007).

${ }^{55}$ R. K. Bryan, Eur. Biophys. J. 18, 165-174 (1990).

${ }^{56} \mathrm{M}$. Jarrell and J. E. Gubernatis, Phys. Rep. 269, 133-195 (1996).

${ }^{57}$ S. Habershon, B. J. Braams, and D. E. Manolopoulos, J. Chem. Phys. 127, 174108 (2007).

${ }^{58}$ E. Rabani, G. Krilov, and B. J. Berne, J. Chem. Phys. 112, 2605-2614 (2000).

${ }^{59}$ A. A. Golosov, D. R. Reichman, and E. Rabani, J. Chem. Phys. 118, 457-460 (2003).
${ }^{60}$ P. D. Miller, Applied Asymptotic Analysis (American Mathematical Society, 2006).

${ }^{61}$ W. H. Miller, J. Chem. Phys. 62, 1899-1906 (1975).

${ }^{62}$ W. H. Miller, Y. Zhao, M. Ceotto, and S. Yang, J. Chem. Phys. 119, 1329-1342 (2003).

${ }^{63}$ J. O. Richardson, Faraday Discuss. 195, 49-67 (2017).

${ }^{64}$ S. Chapman, B. C. Garrett, and W. H. Miller, J. Chem. Phys. 63, 2710-2716 (1975).

${ }^{65}$ A. Lohle and J. Kastner, J. Chem. Theory Comput. 14, 5489-5498 (2018).

${ }^{66}$ R. Korol, N. Bou-Rabee, and T. F. Miller III, J. Chem. Phys. 151, 124103 (2019).

${ }^{67}$ R. Korol, N. Bou-Rabee, and T. F. Miller III, J. Chem. Phys. 152, 104102 (2020).

${ }^{68}$ P. Dierckx, Curve and Surface Fitting with Splines (Oxford University Press, 1995).

${ }^{69}$ E. Jones, T. Oliphant, P. Peterson et al., SciPy: Open source scientific tools for Python (2001).

${ }^{70}$ See https://github.com/greitemann/maxent for the available code kindly provided by Jonas Greitemann.

${ }^{71}$ J. O. Richardson and S. C. Althorpe, J. Chem. Phys. 131, 214106 (2009).

${ }^{72}$ X. Tao, P. Shushkov, and T. F. Miller III, J. Phys. Chem. A 123, 3013-3020 (2019).

${ }^{73}$ M. D. Feit, J. A. Fleck, and A. Steiger, J. Comput. Phys. 47, 412-433 (1982).

${ }^{74}$ D. Neuhauser, M. Baer, R. S. Judson, and D. J. Kouri, Comput. Phys. Commun. 63, 460-481 (1991).

${ }^{75}$ J. C. Tully, J. Chem. Phys. 93, 1061-1071 (1990).

${ }^{76}$ J. E. Lawrence and D. E. Manolopoulos, J. Chem. Phys. 151, 244109 (2019).

${ }^{77}$ Y. Fang, V. P. Barber, S. J. Klippenstein, A. B. McCoy, and M. I. Lester, J. Chem. Phys. 146, 134307 (2017).

${ }^{78}$ R. E. Weston, Jr., T. L. Nguyen, J. F. Stanton, and J. R. Barker, J. Phys. Chem. A 117, 821-835 (2013).

${ }^{79}$ S. Kaufmann, Q. Shuai, D. J. Auerbach, D. Schwarzer, and A. M. Wodtke, J. Chem. Phys. 148, 194703 (2018).

${ }^{80}$ G. A. Voth, D. Chandler, and W. H. Miller, J. Chem. Phys. 91, 7749-7760 (1989).

${ }^{81}$ C. Predescu and W. H. Miller, J. Phys. Chem. B 109, 6491-6499 (2005).

${ }^{82}$ E. Wigner, J. Chem. Phys. 5, 720-725 (1937).

${ }^{83}$ E. Wigner, Trans. Faraday Soc. 34, 29-41 (1938).

${ }^{84}$ M. F. Herman, E. J. Bruskin, and B. J. Berne, J. Chem. Phys. 76, 5150-5155 (1982). 